\title{
An in silico approach to primaquine binding to Trp756 in the external vestibule of sodium channel $\mathrm{Na}_{\mathrm{v}}$ I.4
}

This article was published in the following Dove Press journal:

Journal of Receptor, Ligand and Channel Research

26 July 2011

Number of times this article has been viewed

\author{
Thomas F Scior ${ }^{1}$ \\ Angel A Islas ${ }^{2}$ \\ Evelyn Martínez-Morales ${ }^{2}$ \\ Karina Cuanalo-Contreras' \\ Lourdes Millan-Perezpeña ${ }^{3}$ \\ Eduardo M Salinas- \\ Stefanón ${ }^{2}$
}

'Departamento de Farmacia, ${ }^{2}$ Instituto de Fisiología, ${ }^{3}$ Centro de Química Instituto de Ciencias, Benemérita Universidad Autónoma de Puebla, Puebla, Puebla, México
Correspondence: Eduardo M SalinasStefanón

Instituto de Fisiología, Benemérita Universidad Autónoma de Puebla I4 Sur 630I, CU, San Manuel, Puebla, Puebla CP 72570, México

$\mathrm{Tel}+2222295500$

Fax +222 2295500 ext 730l

Email esalinas@siu.buap.mx
Abstract: The aim of our computed study was to examine the possible binding site of primaquine (PQ) using a combined homology protein modeling, automated docking, and experimental approach. The target models of wild type and mutant types of the voltagedependent sodium channel in rat skeletal muscle $\left(\mathrm{rNa}_{\mathrm{v}} 1.4\right)$ were based on previous work by other researchers. Docking was carried out on the P-loop in the structural model of the $\mathrm{rNa}_{\mathrm{v}} 1.4$ channel, in the open state configuration, to identify those amino acidic residues important for PQ binding. The three-dimensional models of the P-loop segment of wild types and mutant types (W402, W756C, W1239C, and W1531A at the outer tryptophan-rich lip, as well as D400C, E755C, K1237C, and A1529C of the DEKA motif) helped us to identify residues playing key roles in aminoquinoline binding. In good agreement with experimental results, a 1000-fold inhibition loss was observed. Tryptophan 756 is crucial for the reversible blocking effects of PQ. As a result, mutant-type W756C abolished the blocking effect of PQ in voltage-clamp assays.

Keywords: computer docking, homology modeling, $\mathrm{Na}_{\mathrm{v}} 1.4$, sodium channel, oocytes, primaquine $(\mathrm{PQ})$, site-directed mutagenesis, voltage clamp

\section{Introduction}

Under small transmembrane stimulus, voltage-dependent sodium channels produce a large inward current that leads to a uniform depolarization followed by an action potential in many excitable tissues. These channels are important for several processes in tissues, including communication, secretion, or excitation-contraction coupling in cardiac and skeletal muscle cells. They constitute molecular arrangements inside the cell membrane and facilitate the swift passage of millions of ions per millisecond per channel. They cover four different spans, domains DI-DIV, and each has six transmembrane segments (S1-S6). Between segments S5 and S6 of each of the four domains lies the P-segment pore loop of the $\alpha$ subunit that forms the actual pore of the channel. ${ }^{1}$ In this central cylindrical cavity lies the selectivity filter. It has been proposed that at least two rings of amino acid residues delineate this filter motif: DEKA, consisting of aspartic acid, glutamic acid, lysine, and alanine; and EEDD, comprising glutamic acid and aspartic acid. Although the crystal structure of the pore has not yet been elucidated, it is known that the spatial arrangements and the sequence composition of the motif vary according to the specific function of each channel type to filter incoming cations that differ in their ionic charges, radii, and solvation energies. ${ }^{2,3}$

Primaquine (PQ), an 8-aminoquinoline, has been used for several years in the treatment of relapsing malaria produced by Plasmodium vivax and P. ovale. Clinically, PQ shows adverse effects commonly associated with alterations of conduction: depression of 
cardiovascular function, myocardial contractility, and rhythm disturbances. It has been suggested that these effects are related to the blocking of sodium channels ${ }^{4}$ by PQ reversibly binding to the open states. The reverse process of unbinding occurs either when channels move between inactivated states or from an inactivated state to a closed state. Although substantial literature has been developed about the biophysical and pharmacological characteristics of the sodium channel, the effects of several relevant $\mathrm{Na}^{+}$-channel modulators, in particular the aminoquinolines, remain unclear. ${ }^{4}$

The most common binding sites in the pore of sodium channel are the tetrodotoxin (TTX) binding site outside the channel mouth (Y401) and the local anesthetic binding site (LABS) below the DEKA motif at the intracellular mouth (F1579). These sites lie within the pore region and are occupied by specific blockers that decrease the amplitude of the sodium current. ${ }^{5,6}$ The authors of this paper have previously hypothesized that aminoquinolines may use a different binding site inside the sodium channel, distinct from the already known binding sites for certain toxins including TTX, brachotoxin, $\mu$-conotoxins, agatoxin, and local anesthetics, such as lidocaine.

The aim of this study was to examine the possible binding site of PQ using both in silico modeling and direct-site mutagenesis in transfected oocytes from Xenopus laevis. We observed that PQ binds preferentially in the external mouth of the channel, specifically on W756 of DII. Binding to this site was strongly reduced when W756 was mutated to a cysteine residue. In good agreement with experimental results, a 1000fold inhibition loss was observed, suggesting that tryptophan W756 is crucial for the reversible blocking effects of PQ. As a result, the mutated version, $\mathrm{W} 756 \mathrm{C}$, abolished the blocking effect of PQ in voltage-clamp assays.

\section{Methods}

\section{In silico approach}

The target models of wild type (WT) and mutant types (MT) of the voltage-dependent sodium channel in rat skeletal muscle $\left(\mathrm{rNa}_{\mathrm{v}} 1.4\right.$, GeneBank access code \# P15390) were based on the previous work of Tikhonov and Zhorov. ${ }^{7}$ Docking was carried out on the P-loop into the structure model of $\mathrm{rNa}_{\mathrm{v}} 1.4$ channel, in the open state configuration, to identify those amino acidic residues important for PQ binding.

The helical segments and loops of the target sequence that we used were predicted as a consensus line using several offline and web-based secondary structure tools, ${ }^{8-13}$ as well as the ExPASy (Expert Protein Analysis System; Swiss Institute of Bioinformatics, Switzerland) proteomics server.
The helices of the template were displayed using secondary structure profiles and the predicted helical segments of the target were threaded through the secondary structure profile of the template structure. ${ }^{14,15}$ The rationale behind this decision is two-fold: (1) the helical prediction is most reliable because its building rules mostly depend on inner-chain neighborhood atomic interactions and (2) the very low sequence homology where functional coincidence is rarely met (ie, random coincidence of matching target-template pairs cannot be ruled out in the threaded residue pairs). In addition, the identification of the known DEKA motif, with its location and various loop segments between the helices, assisted the manual alignment procedure. Unfortunately, since the template with its open state and pore features is still unique among the crystal channel proteins, neither consensus nor comparative protein modeling is applicable. This lack of alternatives simplifies the model building but at the cost of full dependency on a single backbone. Our structure modeling approach is exhaustively described elsewhere. ${ }^{15}$

Blind docking addresses the problem of finding ligand conformations and positions at the binding site without knowing the final solution provided by X-ray or NMR analyses. Clustering free energies of binding requires sampling ligand orientations according to their receptor affinities (lowest energy scores). The bibliographic and experimental knowledge gained in earlier docking studies (using Insight II [Accelrys, San Diego, CA], Ludi [BASF AG, Central Research, Ludwigshafen, Germany], FlexX [BioSolveIT GmbH, St Augustin, Germany], AutoDock [Molecular Graphic Laboratory, Department of Molecular Biology, Scripps Research Institute, La Jolla, CA], and MOE [Chemical Computing Group, Köln, Germany]) helped us to select the appropriate software. ${ }^{16-19}$

Blind docking was conducted using AutoDock 4 because its calibration set embraces relevant binding patterns seen on alkylaryl moieties such as toluene. ${ }^{20,21}$ Hydrophobic contacts, $\pi$ - $\pi$ bonding, and edge-to-face contacts are expected to play a pivotal role in alkylbenzene binding and hydrogen bonding or ion bridges. ${ }^{22,23}$ The ligand's atomic partial charges were calculated by the Gasteiger approach under A (v ZZ 2.3.2 by Università degli Studi di Milano, Italy) ${ }^{24}$ while the receptor was prepared, ${ }^{25}$ and charges on polar hydrogen atoms added with AutoDock tools. The structure modeling approach for sodium channels is exhaustively described elsewhere. ${ }^{15,26}$

\section{Experimental approach}

Female adult $X$. laevis frogs (Xenopus-I Inc, Dexter, MI) were anesthetized by immersion in $0.2 \%$ tricaine mesylate (MS-222). 
The stage $\mathrm{V}$ and $\mathrm{VI}$ oocytes were surgically removed, placed in OR-2 buffer containing (in mM): $82.5 \mathrm{NaCl}, 2.5 \mathrm{KCl}, 1 \mathrm{MgCl}_{2}$ and 5 HEPES (4-2-hydroxyethylpiperazine-1-ethanesulfonic acid), $\mathrm{pH} 7.6$, and then treated with collagenase $1.3 \mathrm{mg} / \mathrm{mL}$ to remove the follicular membrane. The nuclei of the oocytes were injected by using a nanoliter automatic injector model A203XVY (World Precision Instruments, Sarasota, FL) with 15 to $25 \mathrm{ng}$ of $\mathrm{rNa}_{\mathrm{v}}, \mathrm{W} 756 \mathrm{C}$ and the $\mathrm{rNa}_{\mathrm{v}} 1.4 \alpha$-subunit cDNA clones. ${ }^{27,28}$ Eggs were then maintained at $18^{\circ} \mathrm{C}$ in ND-96 solution containing (in $\mathrm{mM}$ ): $96 \mathrm{NaCl}, 2 \mathrm{KCl}, 1 \mathrm{MgCl}_{2}, 5$ HEPES, and $1 \mathrm{CaCl}_{2}, \mathrm{pH} 7.6$ supplemented with $0.5 \mathrm{mM}$ theophylline, $0.5 \mathrm{mM}$ pyruvate, and $50 \mu \mathrm{g} / \mathrm{mL}$ gentamicin for up to 3 days before recording.

\section{Electrophysiological recording in oocytes}

Oocytes were placed in a $1 \mathrm{~mL}$ recording chamber and continuously superfused with a barium-containing solution at a flow rate of approximately $500 \mu \mathrm{L} /$ minute. Two electrode voltage-clamp recordings were made at room temperature $20^{\circ} \mathrm{C}$ to $22^{\circ} \mathrm{C}$ using an $\mathrm{OC}-725 \mathrm{C}$ amplifier (Warner, New Haven, CT). The electrodes were pulled on a horizontal puller P-97 (Sutter Instruments, Novato, CA). The agarosecushion electrodes, filled with $3 \mathrm{M} \mathrm{KCl}$ (Schreibmayer et $\mathrm{al}^{29}$ ), were used to achieve a final resistance of 0.6 to $1.2 \mathrm{M} \Omega$. The sodium-current signals were digitized at a sampling rate of $10 \mathrm{kHz}$ using an analog-to-digital converter (Digidata 1200; Axon Instruments, Foster City, CA) and stored on a computer for analysis with pClamp software version 8.02 (Axon Instruments, Foster City, CA). The sodium currents $\left(\mathrm{INa}^{+}\right)$were generated by step depolarizations from a holding potential of $-100 \mathrm{mV}$ at $0.1 \mathrm{~Hz}$, unless otherwise indicated. The amplitude of the expressed $\mathrm{INa}^{+}$was typically 1 to $10 \mu \mathrm{A}$. Only oocytes with an $\mathrm{INa}^{+}$peak lower than $7 \mu \mathrm{A}$ were used in the study to minimize voltage-clamp errors. ${ }^{30}$ The current-voltage (I-V) relationships were determined from peak currents generated by 30 millisecond, $10 \mathrm{mV}$ steps from a holding potential of $-100 \mathrm{mV}$ to $+50 \mathrm{mV}$. We used an $\mathrm{EC}_{50}$ of PQ according to the Hill equation: $\mathrm{INa}^{+}=\mathrm{INa}^{+} \max / 1+\left\{\mathrm{EC}_{50} /[\text { primaquine }]^{\mathrm{n}}\right\}$. A concentration of $10 \mu \mathrm{M}$ of PQ was found using this equation in previous work.

\section{Statistics and data analysis}

The results are expressed as mean \pm standard error. The differences between mean data were analyzed with a paired or unpaired Student's $t$-test as appropriate. All the currents were analyzed using the pClamp version 8.02 software (Axon Instruments, Foster City, CA). The graphs were built using
Sigmaplot v. 11 (SPSS Inc, Chicago, Il). The dose-response curve for the PQ effects was adjusted by the Origin 8.02 program (OriginLab Corp, Northampton, MA). A value of $P<0.05$ was used to denote statistical difference between groups.

\section{Results}

PQ, an 8-aminoquinoline, is a potent blocker of voltagegated sodium channels. In Figure 1A, we show the chemical structure of PQ with its molecular electrostatic potential. Figure $1 \mathrm{~B}$ shows the potency of $10 \mu \mathrm{m}$ PQ on the sodiumchannel isoform for the WT (1.4, WT) and the MT (W756C). PQ had a modulating effect on the sodium current in the WT, but the effect was almost abolished in the MT isoform.

We previously demonstrated that W756 is an important residue for binding toluene in $\mathrm{Na}_{\mathrm{v}} 1.4$ channels through hydrophobic attraction. ${ }^{26}$ To find the possible site or sites for PQ binding, we focused on the tryptophan-rich lip area at the outer vestibule. ${ }^{31,32}$ We screened for changes in the PQ blocking of W402C, W756C, W1239C, and W1531A. Those substitutions were less sensitive to the PQ blocking effect, indicating that the outer lip of the channel may play a role in the binding site of the drug. However, the W756C sodium-channel mutation displays a minimal blocking effect at the same drug concentration.

To explore the possible voltage-dependent union for PQ, we plotted the current-voltage relationship for both isoforms. Figure 2 shows the plots generated for five to six different oocytes transfected with WT (A) or MT (B) sodium channel isoforms. The drug has a blocking effect over all the observed range from -40 to $+40 \mathrm{mV}$ with no voltage-dependence observed for the WT. In contrast, the MT has an insignificant blocking effect over all voltage ranges studied. In Figure 2C, we show a cartoon of the sodium-channel model and the relative location of the mutation that produced our PQ-resistant mutant channel isoform.

\section{Docked primaquine in the sodium channel}

To explain the aforementioned experimental findings on a molecular level, we docked PQ into the central pore of $\mathrm{Na}_{\mathrm{v}}$ 1.4. The three-dimensional model generation of the target structure is based on a low homology protein modeling approach using an observed potassium channel pore as its template (pdb id: 1LNQ). ${ }^{26}$ To study side chain flexibility we applied AutoDock 4 and ADTools, which is still a rigid backbone approach. At the outer tryptophan-rich lip we 
A

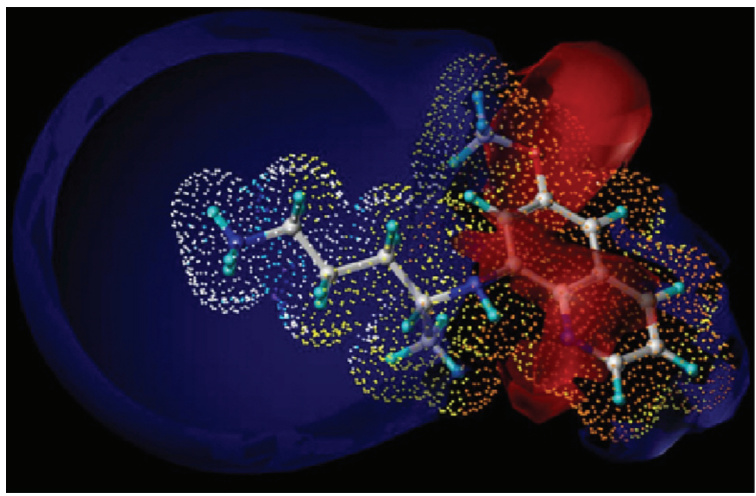

Primaquine
B

WT

W756C
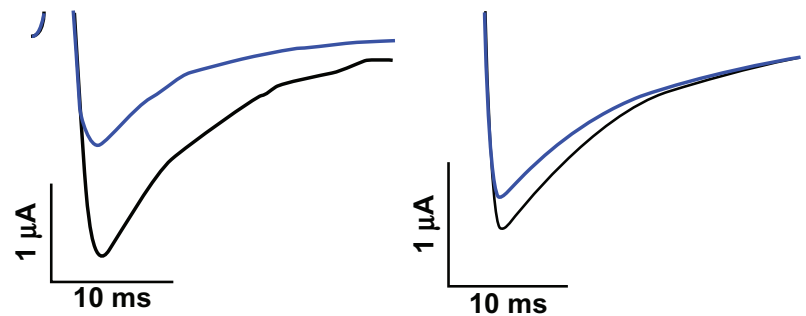

$-100 \mathrm{mv}^{-20}$

$\mathrm{PQ} 10 \mu \mathrm{M}$

Figure I (A) Visualization of atomic lipophilicity projected on the van der Waals surface (colored dots) of primaquine (PQ) (stick model). The electrostatic potential map of $P Q$ is displayed as isocontour plots at $-\mathrm{I}$ and $+10 \mathrm{Kcal} / \mathrm{mol}$. Colors: (i) sticks: white, carbon atoms; dark blue, N; red, O-; light blue, H. (ii) Dotted surface: blue, white, hydrophilic; yellow, orange, greenish: lipophilic. (iii) Blue lobes: positive charges (monocationic ammonium side chain). (iv) Red lobes: negative charges (electronrich aromatic zone). (B) Normalized trace currents obtained from oocytes transfected with $\mathrm{Na}_{\mathrm{v}}$ I.4 wild type (WT), and mutant type, W756C. The blocking effect of PQ on the WT sodium channel was $53 \%$ (left) and the blocking effect of the same drug concentration on the W756C channel was $16 \%$ (right).

A

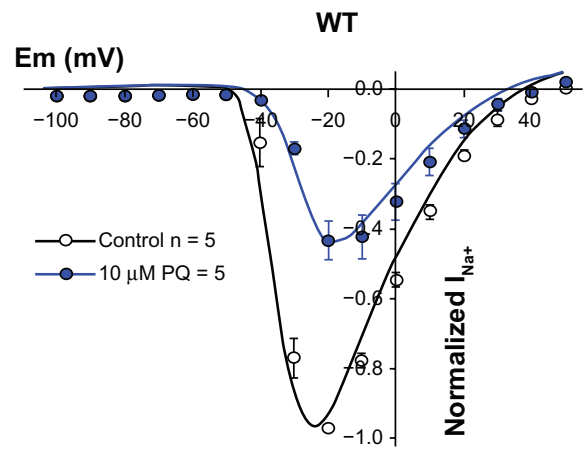

B

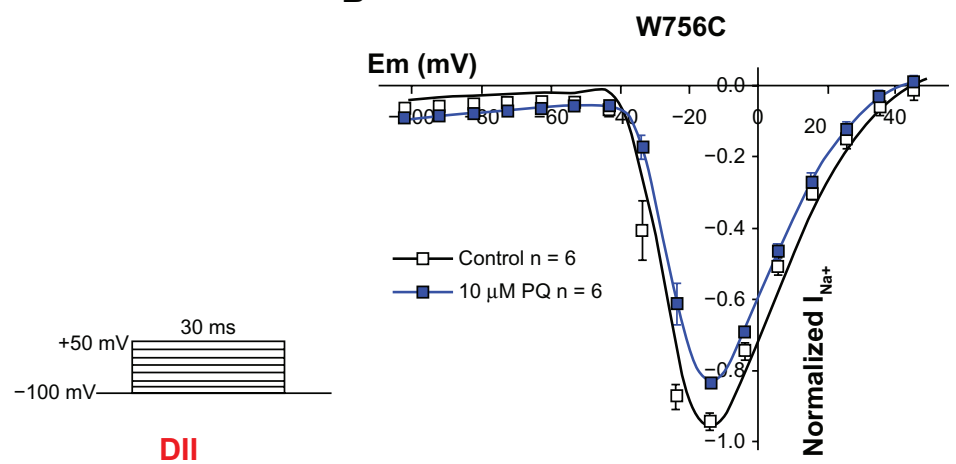

DII

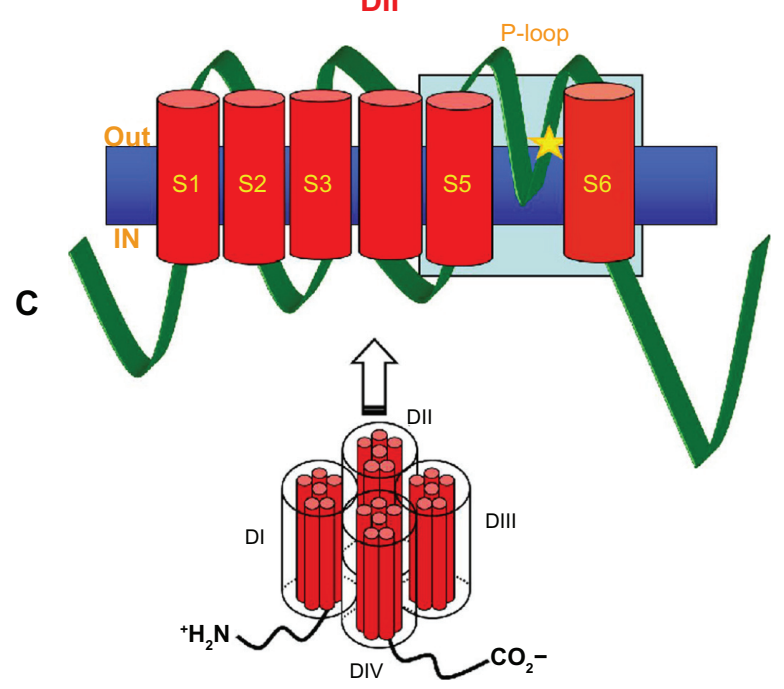

Figure 2 The current-voltage relationship in oocytes transfected with wild type (WT) sodium channels (A) and mutant channels W756C (B). A and B mean ( \pm SEM) voltage relationship of oocytes recorded before and after $10 \mu \mathrm{M}$ of primaquine (PQ). The currents were elicited by step depolarization of $10 \mathrm{mV}$ pulse amplitude, 30 milliseconds duration and stimulation frequency was $0, I \mathrm{~Hz}$, from a holding potential of $-100 \mathrm{mV}$ to $+50 \mathrm{mV}$ (inset). Note that PQ has almost no effect on the mutant type sodium current. (C) Cartoon that represents the topology of the sodium channel and the place where the mutation was made (marked with the star). 
successfully docked PQ into a lipophilic pocket to study the interaction of amino acids with the ligand. The resulting active conformations were clustered according to their orientations by a narrow RMS (root mean square) distance criterion. The most populated solutions for both (WT: 236 out of 256; and MT: 160 out of 256 runs) were also found to be the most favorable energy scores in both cases (WT: $-12 \mathrm{Kcal} /$ mol; and MT: $-6.5 \mathrm{Kcal} / \mathrm{mol})$. The estimated inhibition constant, $\mathrm{Ki}$, for the WT lies in a nanomolar range (best binders $\mathrm{Ki}=1.61 \mathrm{nM}$ ), in stark contrast to MT W756C with a micromolar range (best binders $\mathrm{Ki}=7.44 \mu \mathrm{M}$ ) at room temperature (298 K, Figure 3). Upon conformational rearrangements in the aforementioned pocket, resulting in minor affinity fluctuations, the ligand orients as depicted in Figure 4.

Computationally exploring WT sodium channel, we observed that cationic PQ is trapped within the tryptophanrich outer vestibule (extracellular side) of the channel by hydrophobic and electrostatic interactions, mainly $\pi-\pi$ stacking, edge-to-face contacts, hydrogen bonds and a salt bridge (Figure 4). However, the strong binding of PQ is lost only when changing to the MT W756C. The loss is over 1000-fold, from a nanomolar (WT simulation) to a micromolar level (MT W756C); it is fairly evident that the PQ molecule is rejected out of the pore structure mainly due to the cysteine mutation, thus allowing sodium ions to travel more freely inside the cell (Figure 5).

The tridimensional representation of docked PQ inside the sodium channel is shown in Figure 6B. In Figure 6A, a WT representation of the sodium channel shows the position of the PQ molecule close to the DEKA motif. Interactions with relevant amino acidic residues include aromatic ring stacking, $\mathrm{H}$ bonds and a salt bridge. In Figure 6B, the molecule of PQ

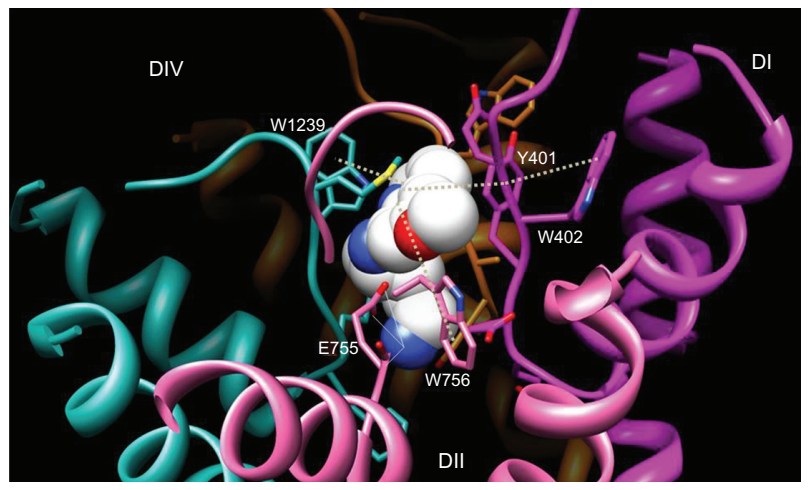

Figure 4 Wild type sodium-channel $\mathrm{Na}_{v}$ I.4 docked with primaquine (PQ) (spheres) Its methyl-butyl ammonium side chain forms a salt bridge with an anionic residue (E755) of the DEKA ring $(1.9 \AA,-0.17 \mathrm{Kcal} / \mathrm{mol}, 2.9 \AA,-0.09 \mathrm{Kcal} / \mathrm{mol})$. W756 on Domain II (in pink), is an important site for binding. Its backbone nitrogen (atom not displayed) forms an $\mathrm{H}$ bond with the PQ's ammonium group $(2.8 \AA,-0.16 \mathrm{Kcal} / \mathrm{mol})$ and its side chain interacts through $\pi$ - $\pi$ stacking with the drug's bicyclic aryl system (4.5 $\AA$ ). WI 239 forms an edge-to-face stacking ( $3.3 \AA$ ) with the PQ molecule and W402 contributes to binding indirectly through $\mathrm{Y} 40 \mathrm{I}$, which also forms a $\pi-\pi$ stacking $(4.5 \AA)$ with the bicyclic aryl system.

is rejected from the DEKA ring by the mutation W756C, decreasing its interaction.

The final representation of PQ docking inside the sodium channel is shown with its electrostatic charge in the proposed binding site, which includes the four domains of the channel with the extracellular side on the top. The PQ goes down close to the DEKA motif. Docking results suggest that PQ binding to mutant $\mathrm{W} 756 \mathrm{C}$ near the selectivity filter remains possible (Figure 6B). Nevertheless the tryptophan to cysteine substitution induces a less favorable binding orientation, in which two significant electronic interactions are lost: a salt bridge with E755 side chain $(-0.17 \mathrm{kcal} / \mathrm{mol})$; and a hydrogen bond with a nitrogen on the backbone of W756 $(-0.16 \mathrm{kcal} / \mathrm{mol})$.
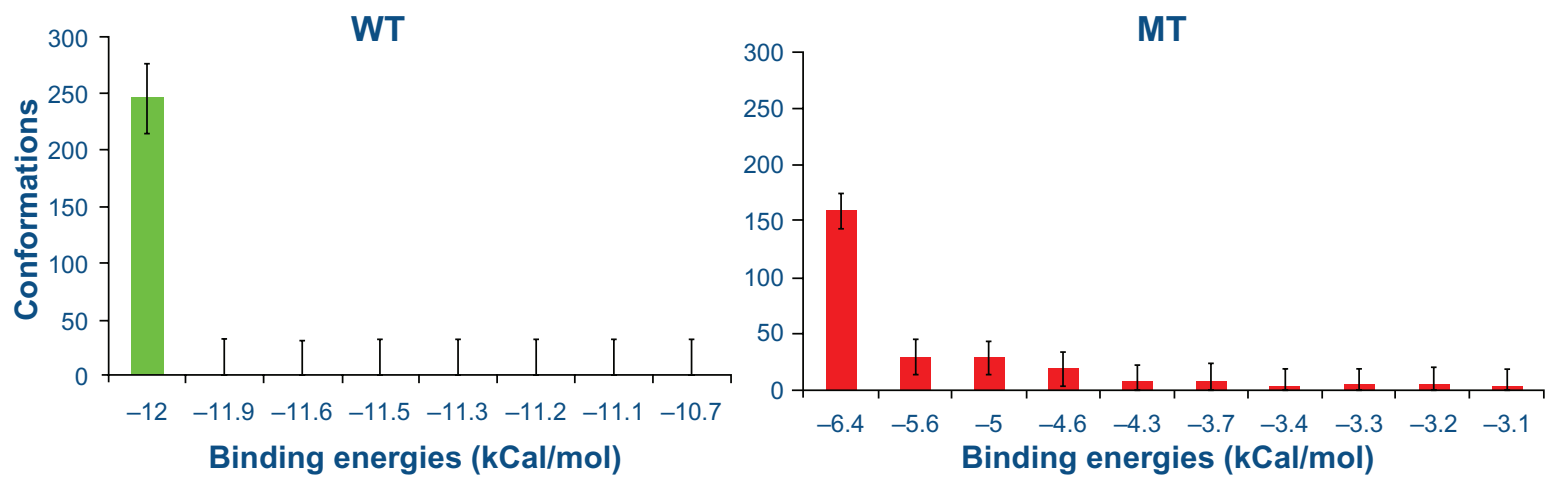

Figure 3 Histograms of docking results. Left side: wild type (WT), right side: mutant type (MT) W756C. The heights of the bars reflect the number of docking hits found with similar docking conformations. In each cluster the overall side chain orientation (inwards or outwards) as well as the general orientation of the pair of cyclohexanes of primaquine were kept in a close range $(\mathrm{RMSD}=2.0)$. In either case, WT and MT, the clusters with the best binders are also the most populated ones, which gave us a consistent picture for interpretation and confidence in our AudioDock 4 modeling.

Abbreviations: SD, standard deviation; RMSD, residual mean SD. 


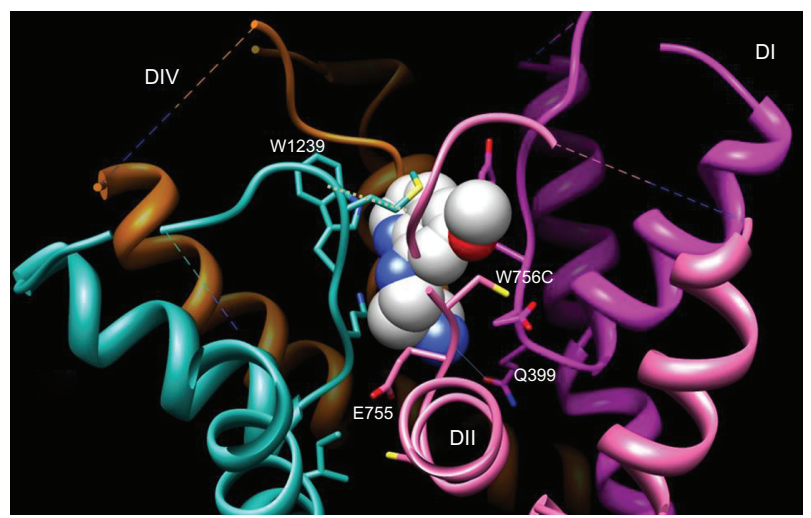

Figure 5 Mutant sodium channel $\mathrm{Na}_{\mathrm{v}}$ I.4 W756C. Cysteine substitution causes a significant reduction in ligand-protein interactions (aromatic and $\mathrm{H}$ bonds), specifically the loss of the salt bridge with $\mathrm{E} 755$ and the $\pi$ - $\pi$ interaction at W756C, on DII (in pink) which accounts for weaker docking energy $(-6.5 \mathrm{Kcal} / \mathrm{mol})$. While WI239 still interacts with the primaquine's bicyclic aryl system forming an edgeto-face stacking $(2.9 \AA$ ) a new H bond has been formed between Gln399 on DI (in magenta) and the drug's ammonium group $(2.2 \AA, 0.12 \mathrm{Kcal} / \mathrm{mol})$.

The slight residual PQ channel block in the mutant form may be explained by the remaining interactions of the drug with other residues such as W1239, primarily an edge-to-face stacking as frequently observed in drug-protein interaction, and also observed in the WT binding orientation. Ultimately the loss of tryptophan 756 side chain entropy-based hydrophobic interactions were responsible for the drop in the calculated total binding energy (Ki) and the consequent experimentally observed drop in current block.

\section{Discussion}

In our in silico and experimental approach to characterizing the mechanism of action of PQ on $\mathrm{Na}_{\mathrm{v}} 1.4$ sodium channels, we found evidence that PQ may bind to a nonpolar aromatic amino acid, a tryptophan in position 756 of domain DII, in the external vestibule. Our experimental results in Figure 1 demonstrate that the sodium current is blocked $52 \%$ by the drug and the substitution of tryptophan with cysteine results in a block of only $15 \%$, probably due to the loss of a $\pi-\pi$ stacking and an $\mathrm{H}-$ bond between PQ and the $\mathrm{W} 756$ residue $(-0.09 \mathrm{kcal} /$ mol). This mutation must have produced an additional global effect resulting in a diminished probability of blocking that was ultimately reflected in the reduction in total binding energy (from $-12 \mathrm{kcal} / \mathrm{mol}$ to $-6.4 \mathrm{kcal} / \mathrm{mol}$, Figure 3 ). However, it was not enough to completely abolish channel blocking (Figure 1). Therefore, in agreement with docking simulations, it is probable that the drug may still weakly interact with other residues, particularly with W1239 on DIII.

Contrary to the widely held belief that blind docking on low homology target proteins goes further than what is reasonable, we see our findings as being in keeping with our ongoing site direct mutagenesis studies including residues such as: D400C, Y401C, W402C (DI), E755C (DII), K1237C, W1239C (DIII) and also A1529C or W1531A (DIV), all of which form the selectivity filter or the outer ring of tryptophans just above the DEKA motif. Those results show that the drug has almost the same blocking effect, except for the K1237C (36\%) and W1531A (39\%).

Figure 7 illustrates the PQ binding site (lateral view), showing how the drug plugs into the channel around a predominantly negative electrostatic charge surrounding the walls of the pore. This position implies that the ammonium side chain would face the DEKA motif and establish a new interaction

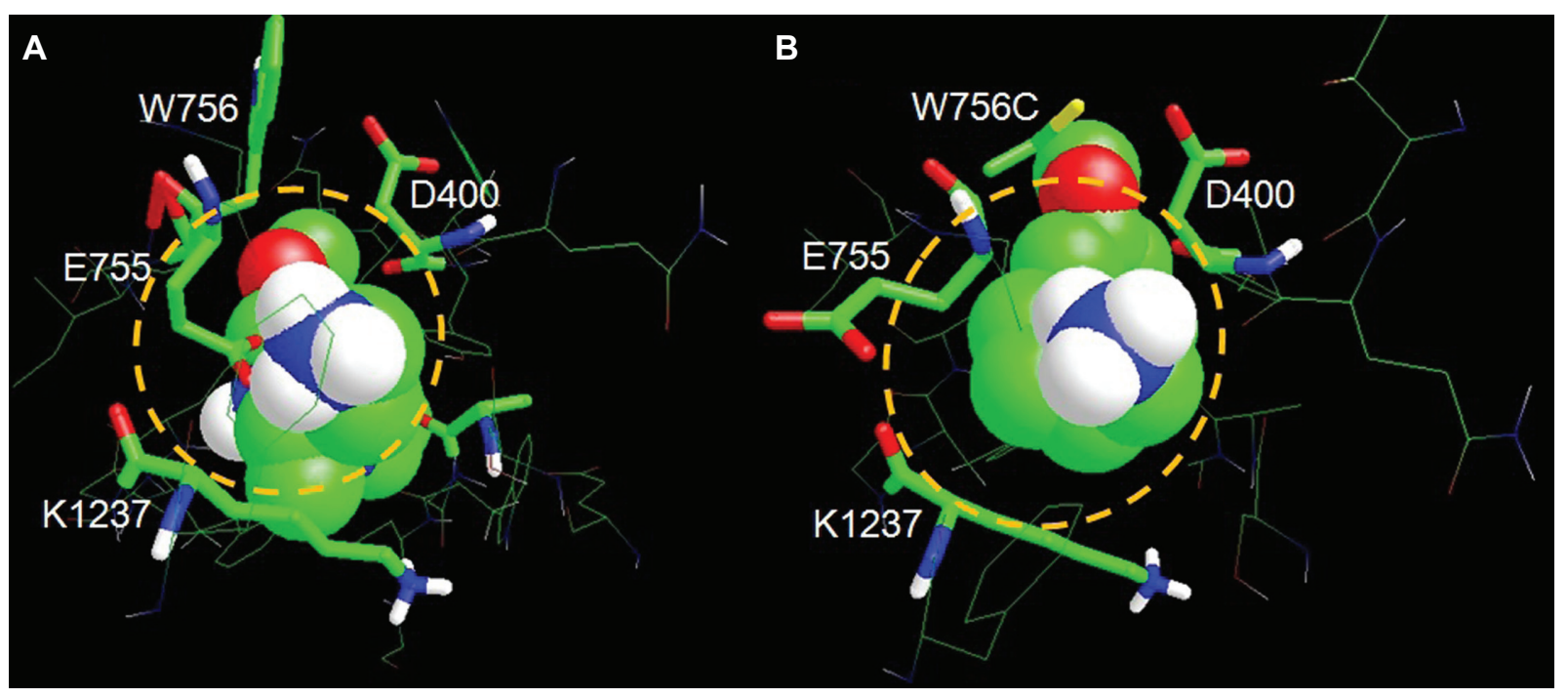

Figure 6 Intracellular view of sodium channel selectivity filter pore. Biologically relevant binding orientations of primaquine (PQ), (A) docked to wild type (WT) channel and (B) to the W756C mutant. Unlike the WT, acidic selectivity filter residue E755 does not allow a salt bridge with the PQ molecule, thus possibly increasing channel pore availability. 


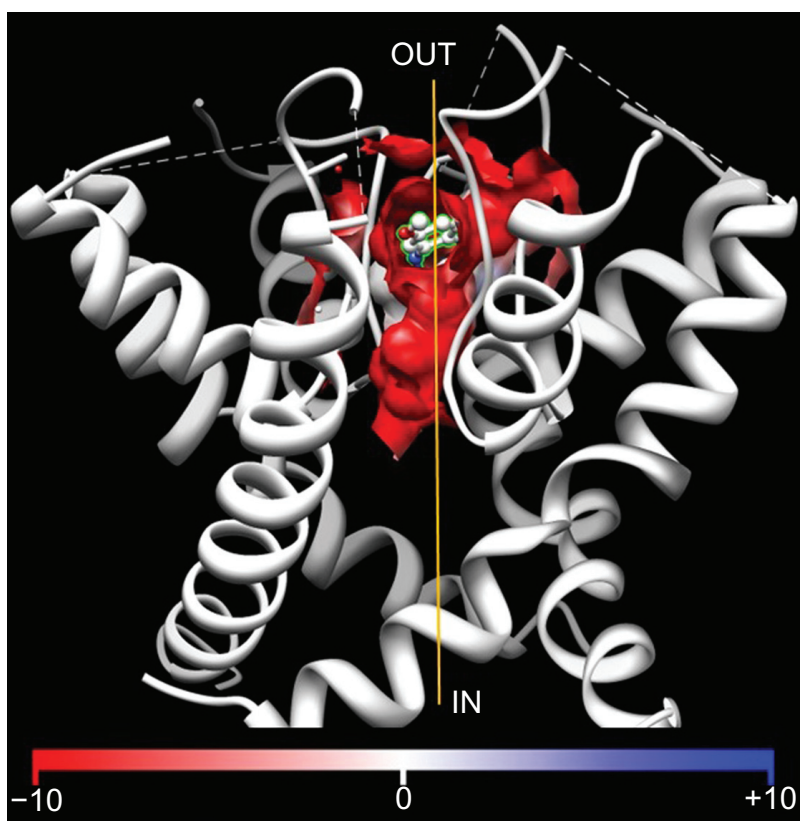

Figure 7 Ribbon representation of wild type (WT) sodium-channel pore in the three-dimensional model of the P-loop segment of the WT sodium channel. Primaquine (PQ) (balls and sticks) is docked into its proposed binding site. Its stretched conformation fills the pore from the tryptophan-rich outer lip (top) towards the dianionic DEKA ring (bottom). The electrostatic surface (5 $\AA$ radius) around the PQ molecule (red) is depicted (coulombic color scale at the bottom).

deep in to the pore, probably with the glutamic acid residue in position 755 and the lysine in position 1237. If this is the case, this new binding site may explain in part the mechanism of action of very well-known toxins like the guanidinium toxins

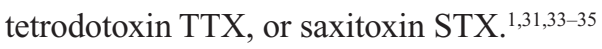

In conclusion, $\mathrm{PQ}$ is an open channel blocker which operates in a dose dependent manner as shown in our previous work on oocyte transfection and cardiac myocytes. ${ }^{5}$ Our tridimensional model contributes to the understanding of how drugs may bind to ion channels, particularly to sodium channels, and proposes an additional insight into the mechanism of action that underlies the collateral effects of PQ as well as other drugs with similar chemical characteristics.

\section{Acknowledgments}

Dr Eduardo Marban kindly provided the $\alpha$-subunit $\mathrm{rNa}_{\mathrm{v}} 1.4$ cDNA vector GW1H (British Biolabs, Oxford, UK). Some of the mutants were kindly provided by Dr Robert Tsushima (York University, Toronto, Canada). Molecular graphics images were produced using the UCSF Chimera ${ }^{36}$ package from the Resource for Biocomputing, Visualization, and Informatics at the University of California, San Francisco, CA (supported by NIH P41 RR001081), and VEGA ${ }^{24}$ ZZ 2.3.2 by Università degli Studi di Milano, Milan, Italy.

\section{Disclosure}

This work was completed with the assistance of the following grants: BUAP-VIEP grant \#SASE-SAL-08-G, and CONACyT \# 48294-M to EMSS, CONACyT \# 2007/526392 to TS, as well as CONACyT PhD Scholarships \# 230292 to KCC, \# 14702 to EMM and \# 242466 to AAI. The authors report no conflicts of interest in this work.

\section{References}

1. Hille B. Ion Channels of Excitable Membranes. 3rd ed. Sunderland Sinauer Associates; 2001.

2. Yamagishi T, Li RA, Hsu K, Marbán E, Tomaselli GF. Molecular architecture of the voltage-dependent $\mathrm{Na}$ channel: Functional evidence for a helices in the pore. J Gen Physiol. 2001;118(2):171-182.

3. Tikhonov D, Bruhova I, Zhorov BS. Atomic determinants of statedependent block of sodium channels by charged local anesthetics and benzocaine. FEBS Lett. 2006;580(26):6027-6032.

4. Orta-Salazar G, Bouchard RA, Morales-Salgado F, Salinas-Stefanon EM Inhibition of cardiac $\mathrm{Na}^{+}$current by primaquine. $\mathrm{Br} J$ Pharmacol. 2002;135(3):751-763.

5. Hanck DA, Makielski JC, Sheets M. Lidocaine alters activation gating of cardiac Na channels. Pflügers Arch. 2000;439(6):814-821.

6. Sunami A, Glaaser IW, Fozzard HA. A critical residue for isorform difference in tetrodotoxin affinity is a molecular determinant of the external access path for local anesthetics in the cardiac sodium channel. Proc Natl Acad Sci U S A. 2000;97(5):2326-2331.

7. Tikhonov DB, Zhorov BS. Sodium channel activators: model of binding inside the pore and a possible mechanism of action. FEBS Lett. 2005;579(20):4207-4212.

8. Gasteiger E, Hoogland C, Gattiker A, et al. Protein identification and analysis tools on the ExPASy server. In: Walker, John M, editor. The Proteomics Protocols Handbook. 1st ed. New York: Humana Press; 2005:571-607.

9. Kabsch W, Sander C. Dictionary of protein secondary structure: pattern recognition of hydrogen-bonded and geometrical features. Biopolymers. 1983;22(12):2577-2637.

10. Jones DT. Protein secondary structure prediction based on positionspecific scoring matrices. J Mol Biol. 1999;292(2):195-202.

11. Cuff JA, Barton GJ. Evaluation and improvement of multiple sequence methods for protein secondary structure prediction. Proteins. 1999;34(4):508-519.

12. Frishman D, Argos P. Incorporation of non-local interactions in protein secondary structure prediction from the amino acid sequence. Protein Eng. 1996;9(2):133-142.

13. Muñoz V, Serrano L. Development of the multiple sequence approximation within the AGADIR model of alpha-helix formation: comparison with Zimm-Bragg and Lifson-Roig formalisms. Biopolymers. 1997;41(5):495-509.

14. Jiang Y, Lee A, Chen J, Cadene M, Chait B, Mackinnon R. Crystal structure and mechanisms of a calcium-gated potassium channel. Nature. 2002;417(6888):515-522.

15. Scior T, Wahab A. Structure prediction of proteins with very low homology: A comprehensive introduction and a case study on aminopeptidase. In: Kaplan, Stanley P, editor. Drug Design Research Perspectives. New York: Nova Science Publishers; 2007:675-708.

16. Coupez B, Lewis RA. Docking and scoring - theoretically easy, practically impossible? Curr Med Chem. 2006;13(25):2995-3003.

17. Stoermer MJ. Current status of virtual screening as analysed by target class. Med Chem. 2006;2(1):89-112.

18. Campbell S, Gold N, Jackson R, Westhead DR. Ligand binding: functional site location, similarity and docking. Curr Opin Struct Biol. 2003;13(3):389-395.

19. McConkey BJ, Sobolev V, Edelman M. The performance of current methods in ligand-protein docking. Curr Sci. 2002;83:845-856. 
20. Morris GM, Goodsell DS, Halliday RS, et al. Automated docking using a Lamarckian genetic algorithm and an empirical binding free energy function. J Comput Chem. 1998;19:1639-1662.

21. Buzko OV, Bishop AC, Shokat KM. Modified AutoDock for accurate docking of protein kinase inhibitors. J Comput Aided Mol Des. 2002;16(2):113-127.

22. Jennings WB, Farrell BM, Malone JF. Attractive intramolecular edge-to-face aromatic interactions in flexible organic molecules. Arch Chem Res. 2001;34(11):885-894.

23. Meyer EA, Castellano RK, Diederich F. Interactions with aromatic rings in chemical and biological recognition. Angew Chem Int Ed Engl. 2003;42(11):1210-1250.

24. Pedretti A, Villa L, Vistoli G. VEGA-an open platform to develop chemo-bio-informatics applications, using plug-in architecture and script programming. J Comput Aided Mol Des. 2004;18:167-173.

25. Gasteiger J, Marsili M. Prediction of proton magnetic resonance shifts: the dependence on hydrogen charges obtained by iterative partial equalization of orbital electronegativity. Org Magn Resonance. 1981;15:353-360.

26. Scior T, Martinez-Morales E, Cruz S, Salinas-Stefanon EM. In silico modeling of toluene binding site in the pore of voltage-gate sodium channel. J Recep Lig Channel Res. 2009;2:1-9.

27. Li HL, Galue A, Meadows L, Ragsdale DS. A molecular basis for the different local ansthetic affinites of resting versus open and inactivated states of the sodium channel. Mol Pharmacol. 1999;55(1):134-141.

28. Nuss HB, Tomaselli GF, Marbán E. Cardiac sodium channels (hH1) are intrinsically more sensitive to block by lidocaine than are skeletal muscle (mu 1) channels. J Gen Physiol. 1995;106(6):1193-1209.
29. Schreibmayer W, Lester HA, Dascal N. Voltage clamping of Xenopus laevis oocytes utilizing agarose-cushion electrodes. Pflugers Arch. 1994;426(5):453-458.

30. Li RA, Tsushima RG, Himmeldirk K, Dime DS, Backx PH. Local anesthetic anchoring to cardiac sodium channels. Circ Res. 1999;85(1):88-98.

31. Tsushima RG, Borges A, Backx PH. Inactivated state dependence of sodium channel modulation by beta-scorpion toxin. Pflügers Arch. 1999;437(5):661-668.

32. Tsushima RG, Li RA, Backx PH. P-loop flexibility in $\mathrm{Na}^{+}$channel pores revealed by single- and double-cysteine replacements. J Gen Physiol. 1997;110(1):59-72.

33. Armstrong CM. Distinguishing surface effects of calcium ion from pore-occupancy effects in $\mathrm{Na}^{+}$channels. Proc Natl Acad Sci U S A. 199;96(7):4158-4163.

34. Hanck DA, Nikitina E, McNulty M, Fozzard HA, Lipkind G, Sheets MF. Using lidocaine and benzocaine to link sodium channel molecular conformations to state-dependent antiarrhythmic drug affinity. Circ Res. 2009;105(5):492-499.

35. Ahern CA, Eastwood AL, Dougherty DA, Horn R. Electrostatic contributions of aromatic residues in the local anesthetic receptor of voltage-gated sodium channels. Circ Res. 2008;102(1):86-94.

36. Pettersen EF, Goddard TD, Huang CC, et al. UCSF Chimera-a visualization system for exploratory research and analysis. J Comput Chem. 2004;25(13):1605-1612.
Journal of Receptor, Ligand and Channel Research

\section{Publish your work in this journal}

The Journal of Receptor, Ligand and Channel Research is an international, peer-reviewed, open access, online journal. The journal welcomes laboratory and clinical findings in the fields of biological receptors, ligands, channel and signal transduction research including: receptors and signalling; ligands; transporters, pores and channels; binding and activation; receptor

\section{Dovepress}

regulation; role of receptors in diseases and their treatment; molecular basis of membrane structure and functions; molecular models of membranes. The manuscript management system is completely online and includes a very quick and fair peer-review system. Visit http://www.dovepress.com/ testimonials.php to read real quotes from published authors. 